$+\operatorname{dim} R$, and that $e_{R}(M)=(n-1+\operatorname{dim} R)$ ! (leading coefficient of $P_{f}(\nu, R)$ ) is a positive integer which depends only on the module $M$. Furthermore, we have shown $[3,4.3]$ that

$$
\chi H_{*}\left(\stackrel{p}{\Lambda}_{f}, R\right)=\left(\begin{array}{l}
n-1 \\
n-p
\end{array}\right) e_{R}(M)
$$

if $f$ is a parameter matrix, i.e., if $m-n+1=\operatorname{dim} R$, where $\chi H_{*}$ stands for the Euler-Poincaré characteristic. We observe that $f$ is a parameter matrix if and only if $I(X(f))$ is an ideal of definition for $\tilde{S}$ generated by a system of parameters, where $S=R\left[X_{11}, \cdots, X_{m n}\right]$ and $\tilde{S}=(S / I(g(m, n)))_{\tilde{m}}$. Therefore, Theorem 2.4 yields immediately

COROLlaRY 2.6. Let $f: R^{m} \rightarrow R^{n}$ be a parameter matrix. Then $e_{R}(M)$ $=e_{\tilde{S}}(\tilde{S} / I(X(f)))$, where $M=$ Coker $f$.

\title{
BIBLIOGRAPHY
}

1. M. Auslander, Modules over unramified regular local rings, Illinois J. Math. 5 (1961), 631-647.

2. D. Buchsbaum, A generalized Koszul complex. I, Trans. Amer. Math. Soc. 111 (1964), 183-196.

3. D. Buchsbaum and D. S. Rim, A generalized Koszul complex. II. Depth and multiplicity, Trans. Amer. Math. Soc., 111 (1964), 197-224.

4. D. G. Northcott, Some remarks on the theory of ideals defined by matrices, Quart. J. Math. Oxford Ser. (2) 14 (1963), 193-204.

BRANDEIS UNIVERSITY

\section{ERRATA, VOLUME 16}

Culbreth Sudler, Jr., A direct proof of two theorems on two-line partitions, pp. 161-168.

Page 165, (3.2). For $a_{\nu}$ read $\alpha_{\nu}$.

Page 167, line -1. For 9531 , read $\begin{array}{llll}11 & 5 & 1 .\end{array}$ 